\title{
Draft Genome Sequence of Neisseria gonorrhoeae Strain NG_869 with Penicillin, Tetracycline and Ciprofloxacin Resistance Determinants Isolated from Malaysia
}

\author{
Geik Yong Ang ${ }^{1}$ Choo Yee Yu ${ }^{1} \cdot$ Delicia Ann Yong ${ }^{1}$ Yuet Meng Cheong ${ }^{2}$. \\ Wai-Fong Yin $^{1} \cdot$ Kok-Gan Chan ${ }^{1}$ (i)
}

Received: 18 October 2015/Accepted: 1 February 2016/Published online: 8 February 2016

(C) Association of Microbiologists of India 2016

\begin{abstract}
Gonorrhea is a sexually transmitted infection caused by Neisseria gonorrhoeae and the increasing reports of multidrug-resistant gonococcal isolates are a global public health care concern. Herein, we report the genome sequence of $N$. gonorrhoeae strain NG_869 isolated from Malaysia which may provide insights into the drug resistance determinants in gonococcal bacteria.
\end{abstract}

Keywords Drug resistance - Genome sequence .

$N$. gonorrhoeae - Sexual transmitted diseases - Gonorrhea

New cases of sexually transmitted infection caused by Neisseria gonorrhoeae were estimated to be a staggering 106.1 million in 2008 [1]. The emergence and dissemination of antimicrobial-resistant $N$. gonorrhoeae is undermining the management and control of gonococcal infections as effective therapeutic options continued to dwindle. Isolation of penicillinase-producing $N$. gonorrhoeae in Malaysia was first reported in 1977 followed by tetracycline- and quinolone-resistant strains in 1990 and 2001, respectively [2-4]. Although the resistance rate for penicillin amongst tested isolates from Malaysia has dropped from $61 \%$ in 2007 to $23.5 \%$ in 2010, resistance

Geik Yong Ang

geikyong@gmail.com

$\triangle$ Kok-Gan Chan

kokgan@um.edu.my

1 Division of Genetics and Molecular Biology, Faculty of Science, Institute of Biological Sciences, University of Malaya, 50603 Kuala Lumpur, Malaysia

2 Jeffrey Cheah School of Medicine and Health Sciences, Monash University Malaysia, 46150 Selangor, Malaysia rate for quinolone remained above $80 \%$ whilst tetracycline was between 35 and $70 \%[1,5]$.

The clinical isolate of $N$. gonorrhoeae strain NG_869 was obtained from a male patient in June 2014 and the genomic DNA was extracted using Epicenter MasterPure DNA Purification Kit (Madison, WI). The genome was sequenced using llumina HiSeq 2000 platform (San Diego, CA) with a 300-bp paired-end library template and a total of 3,251,582 reads with an average length of $98 \mathrm{bp}$ were obtained. De novo assembly performed using CLC Genomics Workbench version 7.0 (Aarhus, Denmark) yielded 220 contigs with an average coverage of 218.13 -fold. The contigs were annotated using Rapid Annotation using Subsystem Technology version 2.0 [6], Prokka [7] and NCBI Prokaryotic Genome Annotation Pipeline version 2.10 [8]. The genome was found to be $99.71 \%$ complete when analyzed using CheckM [9]. The genome size of NG_869 is $2,124,678 \mathrm{bp}$ out of which $2,072,962 \mathrm{bp}$ $(52.6 \% \mathrm{G}+\mathrm{C}$ content) are chromosomal and the remaining $51,716 \mathrm{bp}$ belong to three circular plasmids. A total of 2572 protein coding genes, 346 subsystems and 49 RNA genes ( 3 rRNA and 46 tRNA) were predicted. In the RAST-annotated genome, most of the genes were assigned into amino acids and derivatives (14.8\%) followed by protein metabolism $(12.2 \%)$ and cofactors, vitamins, prosthetic groups and pigment $(10.7 \%)$ subsystems (Table 1). Genes encoding iron acquisition system, multidrug resistance efflux pumps and type IV secretion system were also identified in the genome.

The 42,005-bp plasmid pNG869_1 (47.94\% G + C content) harboured the Dutch-type tetracycline resistance gene (tet $M)$. Another tetracycline resistance gene which was chromosomally mediated was also found as Val-57-toMet point mutation in the ribosomal protein $\mathrm{S} 10$ was identified $[10,11]$. The $b l a_{\mathrm{TEM}-1 \mathrm{~B}}$ gene was found within 
Table 1 Subsystem features of $N$. gonorrhoeae strain NG_869 in RAST annotation system

\begin{tabular}{lr}
\hline Subsystem features & Counts \\
\hline Cofactors, vitamins, prosthetic groups, pigments & 167 \\
Cell Wall and capsule & 102 \\
Virulence, disease and defense & 43 \\
Potassium metabolism & 11 \\
Phages, prophages, transposable elements, plasmids & 4 \\
Membrane transport & 65 \\
Iron acquisition and metabolism & 20 \\
RNA metabolism & 97 \\
Nucleosides and nucleotides & 58 \\
Protein metabolism & 189 \\
Cell division and cell cycle & 31 \\
Regulation and cell signaling & 30 \\
Secondary metabolism & 4 \\
DNA metabolism & 105 \\
Fatty acids, lipids, and isoprenoids & 64 \\
Nitrogen metabolism & 30 \\
Dormancy and sporulation & 1 \\
Respiration & 75 \\
Stress response & 11 \\
Metabolism of aromatic compounds & 30 \\
Amino acids and derivatives & 61 \\
Sulfur metabolism & 30 \\
Phosphorus metabolism & \\
Miscellaneous & \\
\hline & \\
&
\end{tabular}

the 5600-bp African-type $\beta$-lactamase plasmid pNG869_2 (39.65\% $\mathrm{G}+\mathrm{C}$ content) along with strong overlapping promoters $(P a / P b)$ which are associated with an approximate 10 -fold increase in $\beta$-lactamase transcriptional level $[12,13]$. The third plasmid, pNG869_3 $(51.54 \% \mathrm{G}+\mathrm{C}$ content), harboured a virulence-associated protein $\mathrm{D}$ (vapD). Analysis of other genes involved in high-level penicillin resistance revealed that isolate NG_869 possessed the penB resistance determinant with double mutations of Gly-120-to-Asp and Ala-121-to-Gly but the mtrR resistance determinant required by pen $B$ porin mutants to exhibit an increased resistance to penicillin and tetracycline was absent [14]. Furthermore, penicillin-binding protein 2 was not encoded by a mosaic allele and Leu-421-to-Pro substitution was not observed in penicillin-binding protein 1. Mutations in the A subunit of DNA gyrase and ParC subunit of topoisomerase IV which confer resistance to ciprofloxacin were present whereby GyrA possessed the double mutations of Asp-95-to-Ala and Ser-91-to-Phe whilst an Asp-86-Asn mutation was found in ParC [15]. Multilocus sequence typing [16] and N. gonorrhoeae multi-antigen sequence typing [17] revealed that the isolate belonged to ST1587 and ST2575, respectively. To the best of our knowledge, both allelic profiles have not been reported for gonococcal isolates to date. The availability of this genome sequence may provide insights into $N$. gonorrhoeae vaccine development by facilitating the identification of potential vaccine candidates via reverse vaccinology approach [18] and information derived from the plasmids can be used for epidemiologic surveillance of plasmid-mediated antibiotic resistance among $N$. gonorrhoeae isolates [19]. In conclusion, whole-genome sequencing of more $N$. gonorrhoeae isolates would aid in the identification and tracking of resistant determinants in this species and further in-depth comparative genomic analysis will contribute towards understanding the evolution of this pathogen.

Nucleotide sequence accession numbers This Whole Genome Shotgun project has been deposited in GenBank under the Accession No. LFJW00000000. The version described in this paper is the first version, LFJW01000000.

Acknowledgments This work was supported by the High Impact Research Grants, University of Malaya (UM-MOHE HIR Grant UM.C/625/1/HIR/MOHE/CHAN/14/1, No. H-50001-A000027; UMMOHE HIR Grant UM.C/625/1/HIR/MOHE/CHAN/01, No. A000001-50001) awarded to Kok-Gan Chan which are gratefully acknowledged.

\section{References}

1. WHO Western Pacific South East Asian Gonococcal Antimicrobial Surveillance Programmes (2010) Surveillance of antibiotic resistance in Neisseria gonorrhoeae in the WHO Western Pacific and South East Asian regions, 2007-2008. Commun Dis Intell Q Rep 34:1-7

2. Koh CL, Kalaimathee KK, Ngeow YF (1984) Plasmids in penicillinase-producing Neisseria gonorrhoeae in Peninsular Malaysia. Med J Malays 39:269-271

3. Koay AS, My R, Cheong YM (1996) Auxotypes and serogroups of tetracycline-resistant Neisseria gonorrhoeae isolated in Malaysia. J Clin Microbiol 34:1863-1865

4. WHO Western Pacific Region Gonococcal Surveillance Programme (2002) Surveillance of antibiotic resistance in Neisseria gonorrhoeae in the WHO Western Pacific region, 2001. World Health Organization. Commun Dis Intell Q Rep 26:541-545

5. Lahra MM (2012) Surveillance of antibiotic resistance in Neisseria gonorrhoeae in the WHO Western Pacific and South East Asian regions, 2010. Commun Dis Intell Q Rep 36:95-100

6. Aziz RK, Bartels D, Best AA, DeJongh M, Disz T, Edwards RA, Formsma K, Gerdes S, Glass EM, Kubal M, Meyer F, Olsen GJ, Olson R, Osterman AL, Overbeek RA, McNeil LK, Paarmann D, Paczian T, Parrello B, Pusch GD, Reich C, Stevens R, Vassieva O, Vonstein V, Wilke A, Zagnitko O (2008) The RAST server: rapid annotations using subsystems technology. BMC Genom 9:75

7. Seemann T (2014) Prokka: rapid prokaryotic genome annotation. Bioinformatics 30:2068-2069

8. Tatusova T, DiCuccio M, Badretdin MA, Chetvernin V, Ciufo S, Li W (2013) Prokaryotic genome annotation pipeline. In: The 
NCBI handbook (Internet), 2nd ed. NCBI, Bethesda. http://www. ncbi.nlm.nih.gov/books/NBK174280

9. Parks DH, Imelfort M, Skennerton CT, Hugenholtz P, Tyson GW (2015) CheckM: assessing the quality of microbial genomes recovered from isolates, single cells, and metagenomes. Genome Res 25:1043-1055. doi:10.1101/gr.186072.114

10. Pachulec E, van der Does C (2010) Conjugative plasmids of Neisseria gonorrhoeae. PLoS ONE 5:e9962. doi:10.1371/journal. pone.0009962

11. $\mathrm{Hu} \mathrm{M}$, Nandi S, Davies C, Nicholas RA (2005) High-level chromosomally mediated tetracycline resistance in Neisseria gonorrhoeae results from a point mutation in the rpsJ gene encoding ribosomal protein $\mathrm{S} 10$ in combination with the $m t r R$ and penB resistance determinants. Antimicrob Agents Chemother 49:4327-4334

12. Palmer HM, Leeming JP, Turner A (2000) A multiplex polymerase chain reaction to differentiate beta-lactamase plasmids of Neisseria gonorrhoeae. J Antimicrob Chemother 45:777-782. doi:10.1093/jac/45.6.777

13. Lartigue MF, Leflon-Guibout V, Poirel L, Nordmann P, NicolasChanoine MH (2002) Promoters P3, Pa/Pb, P4, and P5 upstream from $b l a_{\mathrm{TEM}}$ genes and their relationship to beta-lactam resistance. Antimicrob Agents Chemother 46:4035-4037. doi:10. 1128/AAC.46.12.4035-4037.2002

14. Olesky M, Zhao S, Rosenberg RL, Nicholas RA (2006) Porinmediated antibiotic resistance in Neisseria gonorrhoeae: ion, solute, and antibiotic permeation through PIB proteins with penB mutations. J Bacteriol 188:2300-2308

15. Deguchi T, Yasuda M, Nakano M, Ozeki S, Ezaki T, Saito I, Kawada Y (1996) Quinolone-resistant Neisseria gonorrhoeae: correlation of alterations in the GyrA subunit of DNA gyrase and the ParC subunit of topoisomerase IV with antimicrobial susceptibility profiles. Antimicrob Agents Chemother 40:1020-1023

16. Larsen MV, Cosentino S, Rasmussen S, Friis C, Hasman H, Marvig RL, Jelsbak L, Sicheritz-Ponten T, Ussery DW, Aarestrup FM, Lund O (2012) Multilocus sequence typing of total-genomesequenced bacteria. J Clin Microbiol 50:1355-1361

17. Martin IM, Ison CA, Aanensen DM, Fenton KA, Spratt BG (2004) Rapid sequence-based identification of gonococcal transmission clusters in a large metropolitan area. J Infect Dis 189:1497-1505. doi:10.1086/383047JID31626

18. Delany I, Rappuoli R, Seib KL (2013) Vaccines, reverse vaccinology, and bacterial pathogenesis. Cold Spring Harb Perspect Med 3:a012476. doi:10.1101/cshperspect.a012476

19. Zheng H, Wu X, Huang J, Qin X, Xue Y, Zeng W, Lan Y, Ou J, Tang S, Fang M (2015) The prevalence and epidemiology of plasmid-mediated penicillin and tetracycline resistance among Neisseria gonorrhoeae isolates in Guangzhou, China, 2002-2012. BMC Infect Dis 15:412. doi:10.1186/s12879-015$1148-9$ 